\title{
Fragmented QRS and Ventricular Dyssynchrony in a Patient Treated with Cardiac Resynchronization Therapy
}

\author{
Stelios Paraskevaidis ", Georgios Giannakoulas, Kostas Polymeropoulos, Vassilios Vassilikos, \\ Emmanouela Dalamanga, Haralambos Karvounis, Stavros Hadjimiltiades and \\ Georgios E. Parcharidis
}

\author{
Cardiology Department, AHEPA University Hospital, Thessaloniki, Greece
}

\begin{abstract}
Cardiac resynchronization is an established treatment modality in patients with heart failure NYHA class III-IV on optimal medical treatment, QRS duration of at least $120-130 \mathrm{~ms}$ and ejection fraction $<35 \%$. Nevertheless, a proportion of patients, up to $20-30 \%$, are considered non-responders as they are not improved by cardiac resynchronization therapy (CRT). This report describes a 63-year-old patient with ischemic cardiomyopathy, severe mitral regurgitation, and fragmented QRS complex in several leads. The patient had LV dyssynchrony identified by echocardiographic tissue Doppler imaging. A biventricular pacemaker was implanted successfully, and resulted in improvement in LV dyssynchrony, LV dimensions, mitral regurgitation and symptoms. Fragmented QRS complex as a marker of ventricular dyssynchrony may play a role in identifying patients who may benefit from cardiac resynchronization therapy.
\end{abstract}

Keywords: Cardiac resynchronization, tissue doppler imaging, fragmented QRS.

\section{BACKGROUND}

Current criteria for cardiac resynchronization therapy (CRT) include heart failure NYHA class III-IV under optimal medical treatment, QRS duration of at least $120-130 \mathrm{~ms}$ and ejection fraction $<35 \%[1,2]$. Ventricular dyssynchrony is demonstrated on the electrocardiogram (ECG) as a bundle branch block, or nonspecific intraventricular conduction delay (QRS $>120 \mathrm{~ms})$ [1-4]. However, echocardiography can better evaluate left ventricular (LV) dyssynchrony compared to QRS duration. Echocardiographic criteria using conventional Doppler imaging were initially used for the assessment of the interventricular dyssynchrony. Recent data have demonstrated that tissue Doppler imaging (TDI) is the optimal method to identify LV dyssynchrony [3,4]. We present a patient with ischemic cardiomyopathy, late QRS activation on surface ECG, and LV dyssynchrony identified by TDI who was successfully treated by CRT.

\section{CASE PRESENTATION}

A 63-year-old male with a prior anterior myocardial infarction, coronary artery bypass graft operation and heart failure NYHA class III despite optimal medical treatment (carvedilol, angiotensin-converting enzyme inhibitors, furosemide, and spironolactone) was evaluated for CRT, in our institution. QRS duration on ECG was $130 \mathrm{~ms}$ with a terminal fragmented portion of QRS complex in leads I, II, III $\mathrm{aVR}, \mathrm{aVL}, \mathrm{aVF}$, and $\mathrm{V}_{3}-\mathrm{V}_{6}$ (Fig. 1). Transthoracic echocardiography showed LV dilatation (left ventricular enddiastolic volume $237 \mathrm{~cm}^{3}$ left ventricular end-systolic volume $160 \mathrm{~cm}^{3}$ ), an ejection fraction of $30 \%$, and severe mitral

*Address correspondence to this author at the Cardiology Department, AHEPA University Hospital, 54637, Thessaloniki, Greece;

Tel: +302310482500; Fax: +302310994673; E-mail: stparask@otenet.gr regurgitation (regurgitant volume $33 \mathrm{ml}$ ). TDI revealed intraventricular dyssynchrony in the left ventricle of $110 \mathrm{~ms}$ between interventricular septum and lateral wall (Fig. 2A). The patient underwent successful implantation of a biventricular pacemaker. The LV lead was placed in the anterolateral vein of the coronary sinus. The R-wave amplitude of the LV pacing lead was $8 \mathrm{mV}$ and the pacing threshold was $1.4 \mathrm{~V}$ at 0.4 ms pulse width. The echocardiographic study performed the next day showed reduction in intraventricular dyssynchrony to $60 \mathrm{~ms}$ and moderate mitral regurgitation (regurgitant volume 23ml) (Fig. 2B). At 4-month follow-up the patient was at NYHA II class and transthoracic echocardiography demonstrated left ventricular end-diastolic volume $201 \mathrm{~cm}^{3}$, left ventricular end-systolic volume $140 \mathrm{~cm}^{3}$ and moderate mitral regurgitation.

\section{DISCUSSION}

Randomized studies with CRT have demonstrated improvement in symptoms, quality of life, ejection fraction, mitral regurgitation, and mortality $[1,2,5]$. In particular, regarding echocardiographic indices, responders to CRT demonstrate a longer LV filling time and reduced total isovolumic time after pacing [6]. However, a proportion of patients are non-responders to CRT, which may be explained by the fact that despite QRS prolongation they do not exhibit LV dyssynchrony. The QRS duration is not a specific and reliable marker of electrical dyssynchrony and has a low predictive value on CRT response [7,8]. One possible reason is that it may exclude abnormal activation patterns; in other words small fragmented portions of the QRS complex may not be considered in the measurement of QRS duration. On the other hand, a number of heart failure patients with narrow QRS are considered responders, as they may demonstrate dyssynchrony [9]. The identification of LV dyssynchrony is an important determinant of responsiveness to CRT. 


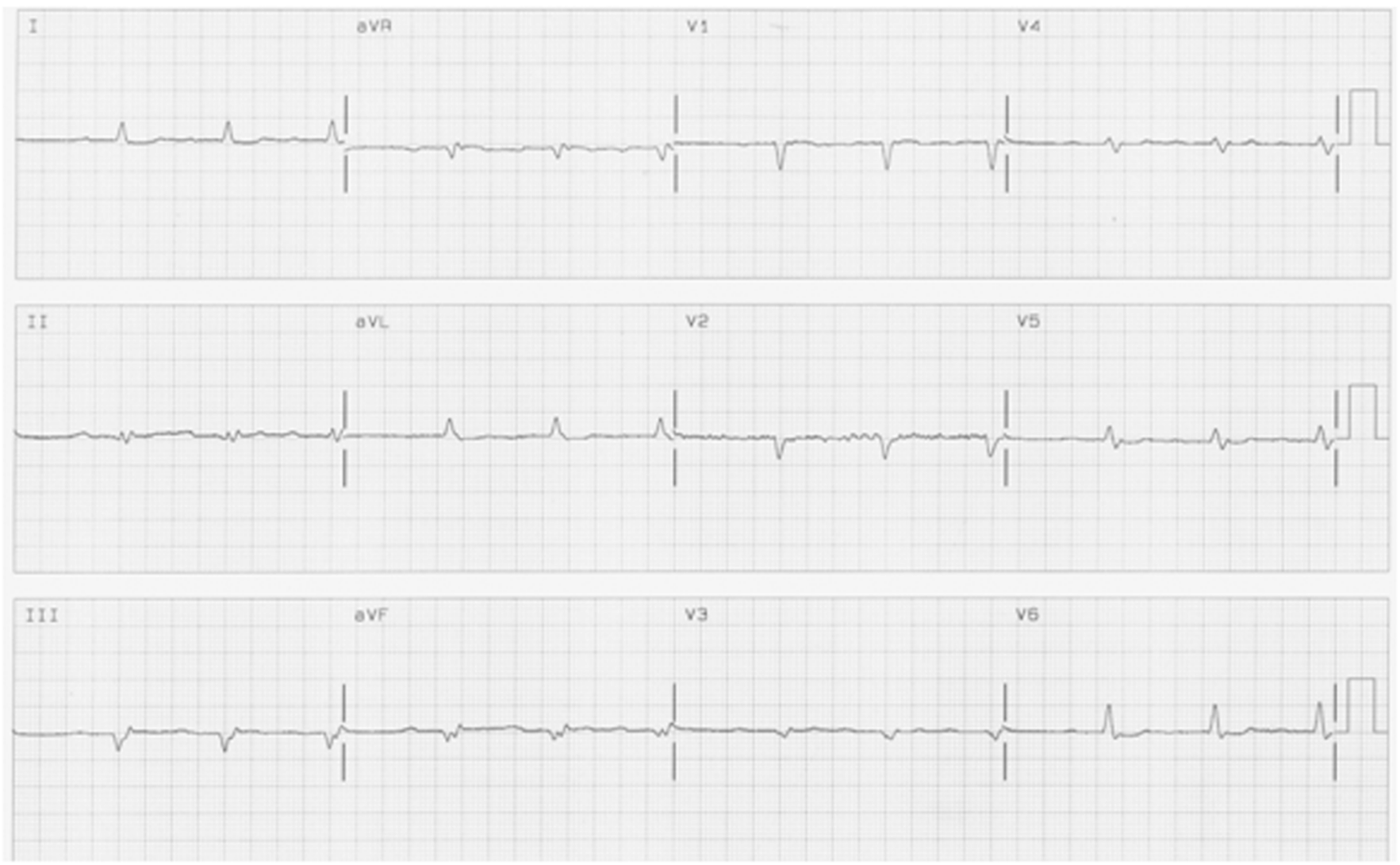

Fig. (1). The 12-lead electrocardiogram before cardiac resynchronization therapy demonstrating a fragmented QRS width of $130 \mathrm{~ms}$ in leads I, II, III aVR, aVL, aVF, and $\mathrm{V}_{3}-\mathrm{V}_{6}$.

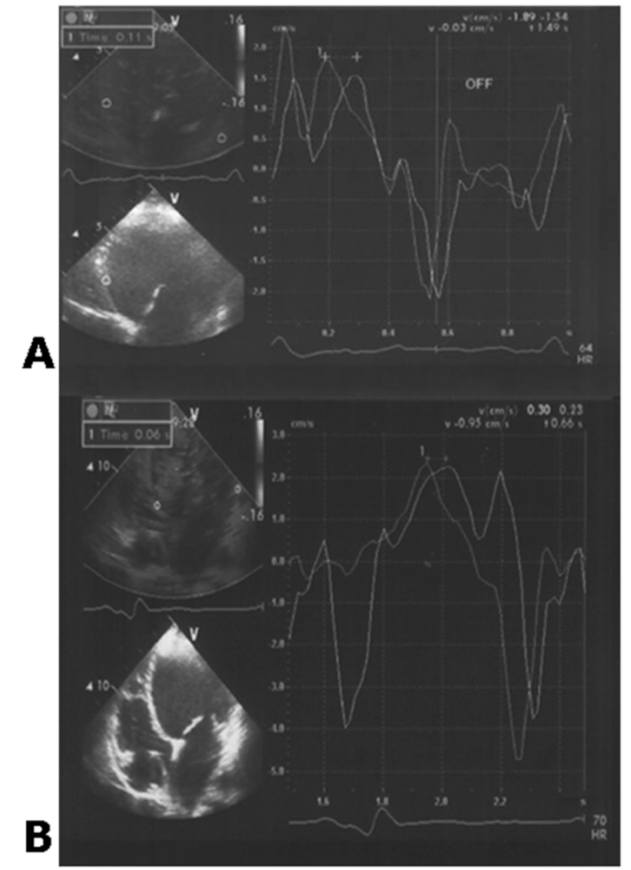

Fig. (2). (A) Echocardiographic image of tissue Doppler tracings before cardiac resynchronization therapy, demonstrating dyssynchrony of $110 \mathrm{~ms}$ between interventricular septum and lateral left ventricular wall. (B) Echocardiographic image of tissue Doppler tracings after cardiac resynchronization therapy, demonstrating 60 $\mathrm{ms}$ delay from interventricular septum to lateral left ventricular wall.
Currently, echocardiographic TDI is the optimal noninvasive method to assess intraventricular dyssynchrony [3,4]. Patients with ischemic cardiomyopathy and LV dyssynchrony may fail to respond to resynchronization, if scar tissue exists around the LV tip pacing lead, despite adequate pacing capture [10].

Three-dimensional electromechanical endocardial mapping of LV was used by Tse et al. to predict successful response in CRT in patients with dilated cardiomyopathy and LBBB [11]. However, this method is invasive and cannot be routinely performed. Another method to identify candidates for CRT using three-dimensional vectorcardiography has been described by Koglek et al. [12]. They used vector ECG analysis to collect information for areas with late electrical excitation and slow or fast depolarization speed.

Fragmented QRS defined as various RSR' and S notching morphologies results in inhomogeneous activation of the ventricles. Its prevalence in patients with chronic heart failure is difficult to establish. In other populations, Pietrasik et al. demonstrated a 53\% prevalence of QRS fragmentation in a cohort of 350 patients diagnosed with a Q-wave MI [13], while Das et al. reported a 27\% prevalence of QRS fragmentation in a cohort of 998 consecutive patients who underwent stress testing [14].

In our case the QRS duration was $130 \mathrm{~ms}$, fragmented QRS was present on surface ECG and LV dyssynchrony of $110 \mathrm{~ms}$ was observed between the interventricular septum and the lateral wall by TDI. CRT was a successful treatment modality for the patient, with a subsequent improvement in 
symptoms and echocardiographic indices. While total QRS duration may not always be a reliable marker of LV dyssynchrony, the fragmented QRS may better reflect LV dyssynchrony. Although fragmented QRS is associated with lower event-free survival for a cardiac event on long-term followup in patients with coronary artery disease [14] it should be evaluated in large clinical studies which will demonstrate the specificity, sensitivity and positive predictive value of this ECG marker in patients with heart failure.

In conclusion, fragmentation (notching) of the QRS complex in a patient with severe ischemic cardiomyopathy was associated with ventricular dyssynchrony confirmed by TDI. This ECG parameter, if present, may serve as a simple marker of ventricular dyssynchrony in patients with heart failure who may benefit from CRT.

\section{CONFLICTS OF INTEREST} est.

The authors declare that they have no conflicts of inter-

\section{FUNDING}

Dr. Giannakoulas has received support from the Hellenic Cardiological Society, the Propondis Foundation and the Cardiological Society of Northern Greece.

\section{REFERENCES}

Abraham WT, Fisher WG, Smith AL, et al. MIRACLE Study Group. Multicenter InSync Randomized Clinical Evaluation. Cardiac resynchronization in chronic heart failure. N Engl J Med 2002; 346: 1845-53.

[2] Linde C, Leclercq C, Rex S, et al. Long-term benefits of biventricular pacing in congestive heart failure: results from the MU1tisite STimulation in cardiomyopathy (MUSTIC) study. J Am Coll Cardiol 2002; 40: 111-8.

[3] Yu CM, Chau E, Sanderson JE, et al. Tissue Doppler echocardiographic evidence of reverse remodeling and improved synchronic- ity by simultaneously delaying regional contraction after biventricular pacing therapy in heart failure. Circulation 2002; 105: 43845 .

[4] Bax JJ, Bleeker GB, Marwick TH, et al. Left ventricular dyssynchrony predicts response and prognosis after cardiac resynchronization therapy. J Am Coll Cardiol 2004; 44: 1834-40.

[5] Cleland JG, Daubert JC, Erdmann E, et al. Cardiac Resynchronization-Heart Failure (CARE-HF) Study Investigators. The effect of cardiac resynchronization on morbidity and mortality in heart failure. N Engl J Med 2005; 352: 1539-49.

[6] Duncan AM, Lim E, Clague J, Gibson DG, Henein MY. Comparison of segmental and global markers of dyssynchrony in predicting clinical response to cardiac resynchronization Eur Heart J 2006; 27 : 2426-32.

[7] Bleeker GB, Schalij MJ, Molhoek SG, et al. Relationship between QRS duration and left ventricular dyssynchrony in patients with end-stage heart failure. J Cardiovasc Electrophysiol 2004; 15: 5449.

[8] Tournoux F, Donal E, Leclercq C, et al. Concordance between mechanical and electrical dyssynchrony in heart failure patients: a function of the underlying cardiomyopathy? J Cardiovasc Electrophysiol 2007; 18: 1022-7.

[9] Achilli A, Sassara M, Ficili S, et al. Long-term effectiveness of cardiac resynchronization therapy in patients with refractory heart failure and "narrow" QRS. J Am Coll Cardiol 2003; 42: 2117-24.

[10] Bleeker GB, Schalij MJ, Van Der Wall EE, Bax JJ. Postero-lateral scar tissue resulting in non-response to cardiac resynchronization therapy. J Cardiovasc Electrophysiol 2006; 17: 899-901.

[11] Tse HF, Lee KL, Wan SH, et al. Area of left ventricular regional conduction delay and preserved myocardium predict responses to cardiac resynchronization therapy. J Cardiovasc Electrophysiol 2005; 16: 690-5.

[12] Koglek W, Brandl J, Oberbichler A, Schmidt K, Grimm G, Butter C. Three-dimensional vectorcardiography to predict CRT-responder. Herzschrittmacherther Elektrophysiol 2006; 17(Suppl 1): I28-36.

[13] Pietrasik G, Goldenberg I, Zdzienicka J, Moss AJ, Zareba W. Prognostic significance of fragmented QRS complex for predicting the risk of recurrent cardiac events in patients with Q-wave myocardial infarction. Am J Cardiol 2007; 100: 583-6.

[14] Das MK, Saha C, El Masry H, et al. Fragmented QRS on a 12-lead ECG: a predictor of mortality and cardiac events in patients with coronary artery disease. Heart Rhythm 2007; 4: 1385-92. 